\title{
Wave absorption control in the new designed photonic metamaterials with artificial opal
}

\author{
Daniela Ionescu, ${ }^{1, *}$ and Gabriela Apreotesei ${ }^{2}$ \\ 1 "Gh. Asachi" Technical University of Iasi, Department of Telecommunications and Informational \\ Technologies, Carol I Blvd. 11, 700506 Iasi, Romania \\ 2 "Gh. Asachi" Technical University of Iasi, Department of Physics, Dimitrie Mangeron Blvd. 67, \\ 700050, Iasi, Romania
}

\begin{abstract}
Photonic metamaterials consisting of artificial opal with magnetic inclusions were considered, used in controllable microwave electronic devices. The analyzed structures consist of matrices of $\mathrm{SiO}_{2}$ nanospheres (diameter $200-400 \mathrm{~nm}$ ) with included clusters of ferrite spinels $\left(\mathrm{Mn}_{\mathrm{x}} \mathrm{Co} 0.6-\mathrm{x} \mathrm{Zn}_{0.4} \mathrm{Fe}_{2} \mathrm{O}_{4}, \mathrm{Ni}_{\mathrm{x}} \mathrm{Co}{ }_{0.6-\mathrm{x}} \mathrm{Zn}_{0.4} \mathrm{Fe}_{2} \mathrm{O}_{4}, \mathrm{La}_{x} \mathrm{Co} 0.6-\mathrm{x} \mathrm{Zn}_{0.4} \mathrm{Fe}_{2} \mathrm{O}_{4}\right.$, $\left.\mathrm{Nd}_{\mathrm{x}} \mathrm{Co}_{0.6-\mathrm{x}} \mathrm{Zn}_{0.4} \mathrm{Fe}_{2} \mathrm{O}_{4}\right)$ in interspherical nanospacing $(4 \div 7 \%$ concentration). The ellipsoidal clusters are polycrystalline, with spatial dimensions of $20-30 \mathrm{~nm}$ and grains of $5-12 \mathrm{~nm}$. A controlled wave absorption was obtained in these high inductivity structures. Evolution of the wave attenuation coefficient, $\alpha[\mathrm{dB} / \mathrm{m}]$, in function of the applied magnetic field and particle inclusion size, for different content of the magnetic ions in the ferrite inclusion, have been determined at frequencies around the samples ferromagnetic resonance, by structural simulation. The test configuration was: sample inside the rectangular waveguide, mode $T E_{10}$, in the frequency range $24 \div 40 \mathrm{GHz}$. The polarizing magnetic field for the ferrites was tested in the range of $0 \div 20 \mathrm{kOe}$ and minimized by modifying the structure. The metamaterial design optimization was realized, controllable by different parameters at structure level. The ferromagnetic resonance influence on the control process was pointed out and also the particular results and effects which can be induced by the resonant behavior.
\end{abstract}

\section{Introduction}

Electromagnetic wave propagation through the new photonic metamaterials occurs in special conditions, considering the periodic variation of the dielectric constant or refraction index in these nanomaterial structures [1]. A large area of application characterizes these metamaterials, because the structures can be adjusted and controlled, in the fields of optoelectronics, communications, sensing devices and biological devices, and also energy storage (thermophotovoltaics, electrochromic and resonant processes, etc.) [2, 3]. We have studied here photonic metamaterials consisting of artificial opal with magnetic inclusions, used in controllable microwave electronic devices like attenuators, phase shifters, etc.

\footnotetext{
*Corresponding author: danaity@yahoo.com
} 


\subsection{Artificial opal in photonic metamaterials - special properties}

The analyzed structures consist of matrices of $\mathrm{SiO}_{2}$ nanospheres (diameter 200 to $400 \mathrm{~nm}$ ) with included clusters of ferrite spinels in interspherical nanospacing $(4 \div 7 \%$ concentration). The ellipsoidal clusters in the inter-sphere voids are in polycrystalline phase. Structure properties depend on ferrite nature, concentration of the magnetic ions in the ferrite, ferrite lattice properties, grain dimensions and dimensions of the polycrystalline inclusions and, in the same time, depend on the nanosphere voids dimensions and the applied fields.

High inductivity structures were obtained and the ferrite inclusions confer to the nanocomposite properties like high resistivity, low dielectric loss, high Curie temperature, chemical stability $[2,4]$.

A ferromagnetic resonance occurs, depending on the inclusions nature and linked by the magnetic polarizing field $H_{=}$. By studying the material behavior close to resonance, some maxima and minima of the material properties can be pointed out, occurring at particular values of the physical and geometrical parameters of the samples or of the applied fields.

\subsection{Theoretical considerations}

We have to consider that the attenuation, $\alpha$, of the electromagnetic waves propagating in a lossy medium is given by the expression [2]:

$$
\alpha=\omega \sqrt{\frac{\mu^{\prime} \varepsilon^{\prime}}{2} \cdot \sqrt{-1+\frac{\mu^{\prime \prime}}{\mu^{\prime}} \cdot \frac{\varepsilon^{\prime \prime}+\sigma / \omega}{\varepsilon^{\prime}}+\sqrt{1+\left(\frac{\mu^{\prime \prime}}{\mu^{\prime}}\right)^{2}+\left(\frac{\varepsilon^{\prime \prime}+\sigma / \omega}{\varepsilon^{\prime}}\right)^{2}+\left(\frac{\mu^{\prime \prime}}{\mu^{\prime}} \cdot \frac{\varepsilon^{\prime \prime}+\sigma / \omega}{\varepsilon^{\prime}}\right)^{2}}}}
$$

where the wave pulsation $\omega=2 \pi f ; f$ is the frequency; $\varepsilon=\varepsilon^{\prime}+\mathrm{j} \cdot \varepsilon$ "' is the electric permittivity of the medium; $\mu=\mu^{\prime}+\mathrm{j} \cdot \mu^{\prime \prime}$ is the magnetic permeability; $\mathrm{j}$ is the imaginary unit and $\sigma$ is the material conductivity. The effective permittivity and permeability of the metamaterial samples can be determined and varied with help of the 3D simulator.

The total wave attenuation can be written as:

$$
\text { Atenuation }[d B]=\alpha[d B / m] \cdot l[m]
$$

Where the wave attenuation coefficient, $\alpha[\mathrm{dB} / \mathrm{m}]$ has to be multiplied with the sample length $l$ along the wave propagation direction. Attenuation dependence will be studied in function of different parameters and also close to the ferromagnetic resonance.

The ferromagnetic resonance frequency (FMR) for metamaterial samples can be estimated with Kittel's formula [2,5]:

$$
f_{0[S I]}=\gamma \sqrt{\left(H_{=}+H_{a}\right) \cdot\left(H_{=}+H_{a}+M_{S}\right)}
$$

Where $M_{S}$ represents the saturation magnetization, $H_{a}=$ the anisotropy field and $\gamma=2.8$ $\mathrm{GHz} / \mathrm{kOe}=$ the gyromagnetic ratio. The external bias magnetic field $H_{=}$was taken in range of $0 \div 20 \mathrm{kOe}$ in the case of the analyzed metamaterial samples with ferrite inclusions.

\section{Wave absorption control - results and discussions}

Analysis of the artificial opal with magnetic inclusions was performed by structural simulation method, using the HFFS program. The internal structure was reproduced with 
help of the 3D simulation tool, based on the real lattice model (Figure 1). The composed metamaterial structure consists of matrices of $\mathrm{SiO}_{2}$ nanospheres (diameter 200 to $400 \mathrm{~nm}$ ) with included clusters of ferrite spinels in interspherical nanospacing $(4 \div 7 \%$ concentration). The ellipsoidal clusters in the inter-sphere voids are in polycrystalline phase, with spatial dimensions of $20-30 \mathrm{~nm}$ and grains of 5-12 nm. Spinel combinations $\mathrm{Mn}_{\mathrm{x}} \mathrm{Co}_{0.6-\mathrm{x}} \mathrm{Zn}_{0.4} \mathrm{Fe}_{2} \mathrm{O}_{4}, \quad \mathrm{Ni}_{\mathrm{x}} \mathrm{Co}_{0.6-\mathrm{x}} \mathrm{Zn}_{0.4} \mathrm{Fe}_{2} \mathrm{O}_{4}, \quad \mathrm{La}_{\mathrm{x}} \mathrm{Co}_{0.6-\mathrm{x}} \mathrm{Zn}_{0.4} \mathrm{Fe}_{2} \mathrm{O}_{4}, \quad \mathrm{Nd}_{\mathrm{x}} \mathrm{Co}_{0.6-\mathrm{x}} \mathrm{Zn}_{0.4} \mathrm{Fe}_{2} \mathrm{O}_{4}$ spinels have been considered.

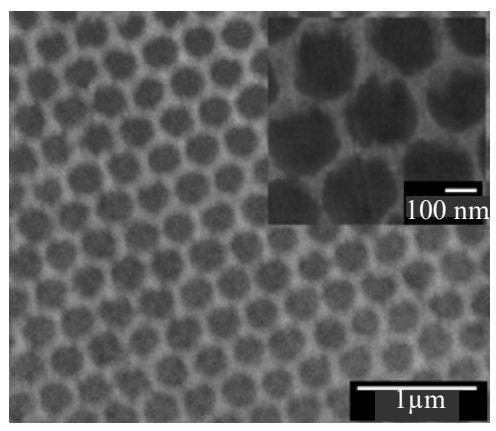

a)
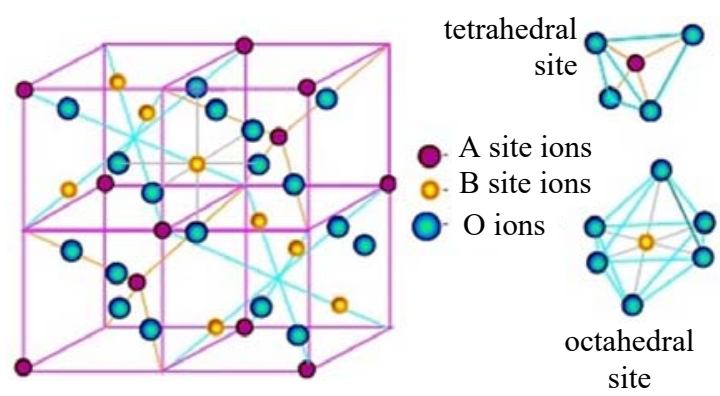

b)

Fig. 1. a) The 3D structure of the 111 face of the silicon opal photonic crystal (after Kurdyukov \&, 2017, Springer, ISBN 3319564226); b) the spinel structure (source: All 'Bout Chemistry, http://www.chemohollic.com/).

The spinel structure $\left(\mathrm{AB}_{2} \mathrm{O}_{4}\right)$ is essentially cubic, with the $\mathrm{O}^{-}$ions placed in an fcc lattice. The metallic cations occupy $1 / 8$ of the tetrahedral sites and $1 / 2$ of the octahedral sites. The unit cell presents $32 \mathrm{O}^{-}$ions [4]. We have considered $\mathrm{Fe}$ ions in the $\mathrm{B}$ sites and the A sites are occupied by magnetic $(\mathrm{Co}, \mathrm{Ni})$ or non-magnetic ions $(\mathrm{Mn}, \mathrm{Zn}, \mathrm{La}, \mathrm{Nd})$, in order to obtain spinels with different properties for analysis.

\subsection{Simulation set-up}

The test configuration of the metamaterial samples is presented in Figure 2.

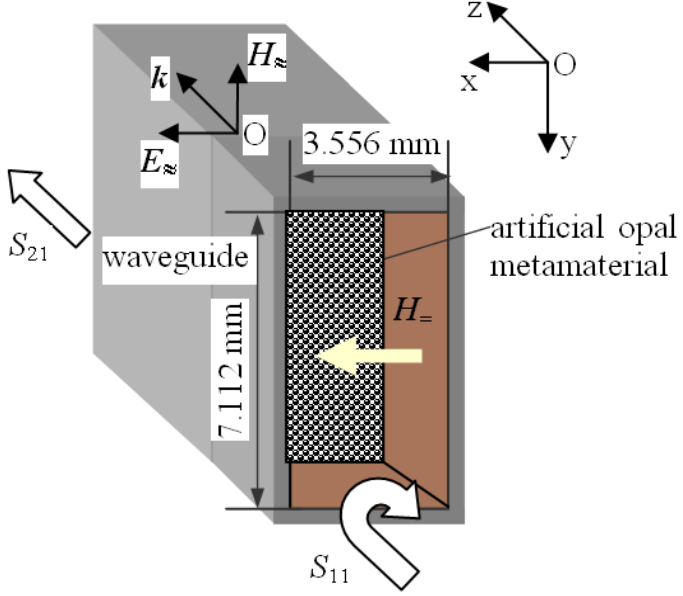

Fig. 2. WR-28 Waveguide with material sample, operating mode $T E_{10}$. 
Sample is placed inside the rectangular waveguide (WR-28, $a \times b=7.112 \times 3.556 \mathrm{~mm}$ ), mode $T E_{10}$, in the frequency range $24 \div 40 \mathrm{GHz}$ ( $\mathrm{Ka}$ band). The bias magnetic field $H_{=}$for the ferrites is in the sample plane, parallel to the microwave electric field $E_{\approx}$. The microwave field propagates transverse of the metamaterial sample, which fills the crosssection of the waveguide and has a thickness $d$. The polarizing continuous field $H_{=}$, was considered in the range of $0 \div 20 \mathrm{kOe}$. The task of minimizing the $H=$ value in order to control and diminish the wave absorption in metamaterial was an objective of study. Structure modification and exploiting the resonance vicinity have been the methods of work.

\subsection{Results for the wave absorption in metamaterial}

A controlled electromagnetic wave absorption was obtained in these high inductivity structures. Evolution of the electromagnetic wave attenuation coefficient, $\alpha[\mathrm{dB} / \mathrm{m}]$, in function of the applied magnetic field and particle inclusion size, at frequencies around the samples ferromagnetic resonance have been determined by structural simulation and represented on graphs. Parametrical curves have been obtained for the frequencies: 24, 28, 32,36 , and $40 \mathrm{GHz}$. Graph for the attenuation coefficient, $\alpha[\mathrm{dB} / \mathrm{m}]$, in function of the applied magnetic field $H_{=}$, for samples with different content of the magnetic ions (Co in particular) in the ferrite inclusion are given in Figure 3.

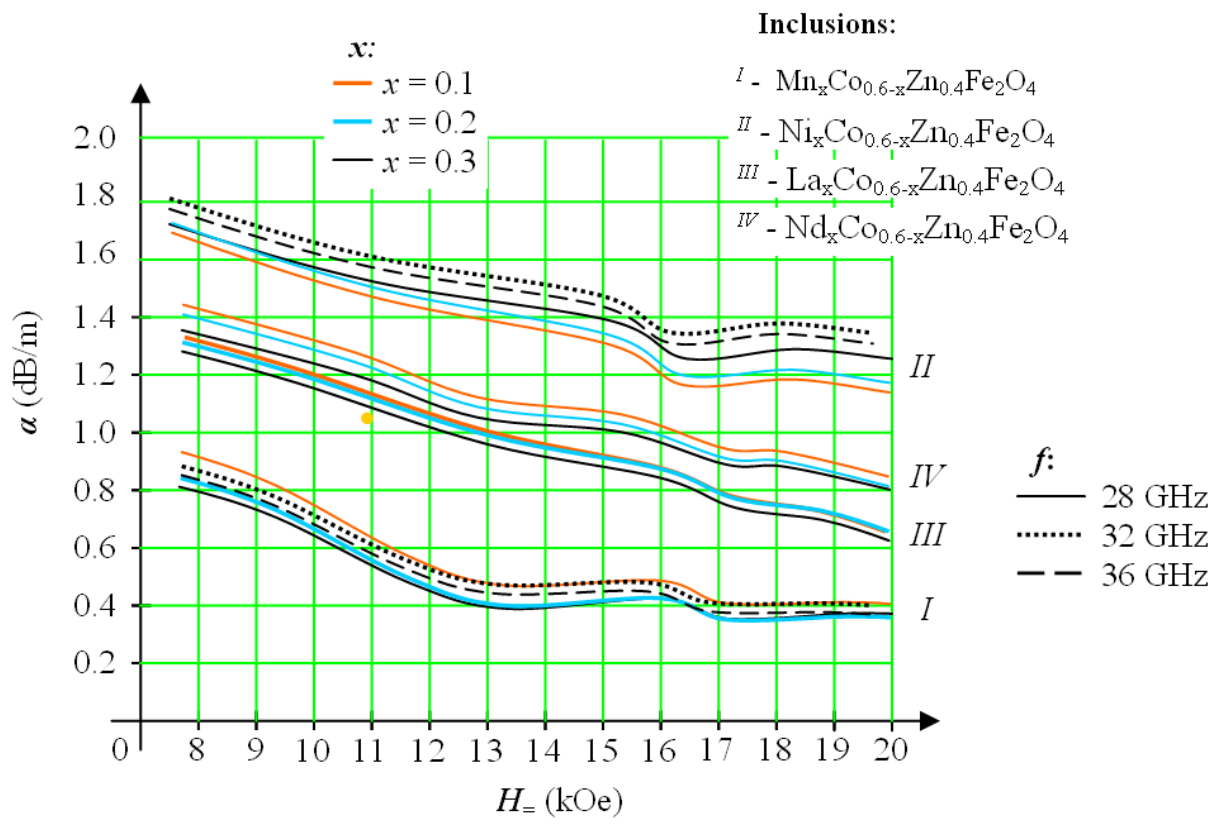

Fig. 3. Wave attenuation coefficient dependence on the applied magnetic field $H=$, for different content $x$ of the magnetic ions in the ferrite inclusion. Particular curves evolution with frequency was indicated on graph.

One observes that the materials having ferrite inclusions with two magnetic ions in the structure $(\mathrm{Ni}, \mathrm{Co})$ present the highest attenuation of the electromagnetic wave, due to the stronger interactions between the internal and the crossing field. In the same time, at the same category of samples, by varying the magnetic ions content in ferrite, we can vary the attenuation. 
But, when the attenuation minimum shifts at lower bias fields, the attenuation increases. Lower attenuation values can be obtained for inclusion ferrites with lower magnetic ions content, having different paramagnetic ions in the formulae ( $\mathrm{Nd}, \mathrm{La}$, and $\mathrm{Mn})$.

Ferromagnetic resonance for the material samples occurs between 32 and $36 \mathrm{GHz}$, as is was reported in literature $[2,4]$. In this frequency region, the normal decreasing variation of the attenuation coefficient with $H_{=}$field presents tendencies of minima, indicated by simulation method, which cannot be described by theoretical calculation. The position of these minima helps us to make correlation between the structural parameters in order to minimize the necessary bias field for the ferrite. Graph for the attenuation coefficient, $\alpha$ $[\mathrm{dB} / \mathrm{m}]$, in function of the particle inclusion size (ellipsoidal polycrystalline inclusions long axis) are given in Figure 4.

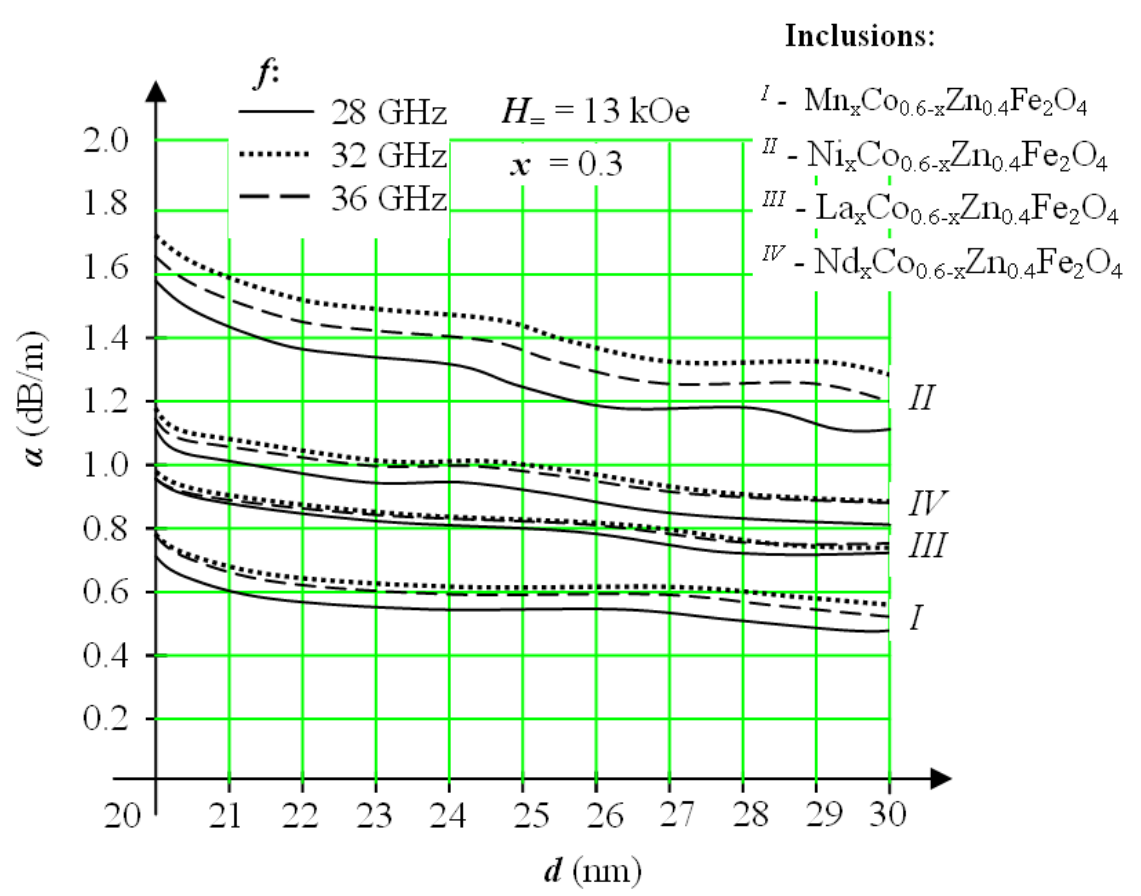

Fig. 4. Evolution of the wave attenuation coefficient $\alpha[\mathrm{dB} / \mathrm{m}]$, in function of the particle inclusion size. Curves are parametrical, represented for different operating frequencies in the resonance frequency region.

A slow decreasing with exponential allure for propagating wave attenuation, when inclusion particles are bigger, can be observed, which can be mathematically described. At frequencies over the ferromagnetic resonance a tendency of more accentuate decreasing of attenuation can be evidenced, in comparison with the theory predictions of continuous exponential decay. We have to notice that we can count on this accentuated decay only at frequencies superior to the resonance. Lower frequency waves (in the considered interval) are attenuated more because wave interaction processes with internal structure (like the multiple Bragg diffraction and scattering [6]) occur preponderant at these frequencies.

\section{Properties control - discussions and conclusions}

Variation of the content of the magnetic ions in the ferrite inclusion influences the characteristics of the parametrical curves. Wave attenuation generally increases in the case 
of inclusions with high magnetic moment. Paramagnetic ions in the inclusion ferrite can decrease the wave attenuation, by decreasing the magnetic moment which interacts with the propagating field $[7,8,9$, and 10]. Structures have been designed in order to obtain a bias magnetic field for the ferrite inclusions as low as possible. The attenuation can be controlled with help of this polarizing magnetic field. The ferromagnetic resonance influence (occurring in the interval of $32-36 \mathrm{GHz}$, depending on the inclusions) on the metamaterial control process was pointed out and also the particular results and effects which can be induced at frequencies close to the resonance.

Our simulation method indicates a strategy for design optimization, in the case of the magnetic metamaterials structured like 3D-nanocomposites, controllable by different parameters at structure level. The presence of the ferromagnetic resonance influences the control process of the metamaterial performances, especially at frequencies close to the resonance. The polarizing magnetic field for the ferrites inclusions can be minimized by modifying the structure, also considering these effects due to the metamaterial particular behavior in conditions close to resonance occurring. The controlled metamaterials consisting of artificial opal with ferrite nanometric inclusions can be used at the attenuators, phase shifters and other devices designed for millimeter wave applications. They can be included in the category of the high frequency devices, manufactured with novel nanotechnologies, controlled by a magnetic field.

\section{References}

1. M. Samoylovich, A. Rinkevich, A. Belyanin, Absorption of electromagnetic waves of millimeter waveband in metamaterials based on opal matrices, $10^{\text {th }}$ Int. Conf. - The Experience of Designing and Application of CAD Systems in Microelectronics, 2009

2. Y. Hao, R. Mittra, FDTD Modeling of Metamaterials. Theory and Applications (Artech House, 2009)

3. F. Capolino, Applications of Metamaterials (CRC Press, 2017)

4. X. C. Tong, Photonic Metamaterials and Metadevices. In: Functional Metamaterials and Metadevices. Springer Series in Materials Science, Vol. 262. Springer, Cham, pp. 71-106 (2018)

5. S. Cabrini, S. Kawata, Nanofabrication Handbook (CRC Press, 2012)

6. A. V. Moroz, M. Limonov, M. Rybin, K. Samusev, Multiple Bragg Diffraction in Low-Contrast Photonic Crystals Based on Synthetic Opals, Physics of the Solid State 53, pp. 1105-1113 (2011)

7. M. V. Vasnetsov, V. Yu. Bazhenov, V. V. Ponevchinsky, A. D. Kudryavtseva, N. V. Tcherniega, Linear and Nonlinear Optics of Synthetic Opal, 2016 IEEE $7^{\text {th }}$ Int. Conf. on Advanced Optoelectronics and Lasers, Sept. 12-15, Odessa, Ukraine (2016)

8. V. Klimov, Nanoplasmonics, Cap. 11, (CRC Press, 2014)

9. A. A. Eliseev, D. F. Gorozhankin, K. S. Napolskii, et all., Determination of the Real Structure of Artificial and Natural Opals on the Basis of Three Dimensional Reconstructions of Reciprocal Space, JETP Letters 90(4), pp. 272-277 (2009)

10. A. Lavrinenko, R. J. Leyrer, W. Wohlleben, N. Dissaux, K. Heggarty, M. Boyle, R. Kivan, A. Neumeister, Progress in photonic metamaterials based on artificial opals, Int. Conf. on Coherent and Nonlinear Optics and Int. Conf. on Lasers, Applications, and Technologies, Minsk, Belarus, May (2007) 